\title{
THE IDENTIFICATION OF A FLUORESCENT REDUCING SUBSTANCE IN THE URINE OF PATIENTS WITH RHEUMATOID ARTHRITIS

\author{
THE EXCRETION OF 3-HYDROXYANTHRANILIC ACID IN THIS \\ AND OTHER CONDITIONS
}

\author{
BY \\ MARY McMILLAN* \\ From the Department of Clinical Chemistry, Edinburgh Royal Infirmary
}

(RECEIVED FOR PUBLICATION SEPTEMBER 7, 1959)

The report that 2,5-dihydroxyphenylpyruvic acid occurs in the urine of patients with collagen disease has not been confirmed, and no evidence was found for regarding collagen disease as being due to an inherent error in tyrosine metabolism as suggested by Japanese workers.

A strongly reducing substance was conspicuous in the urine of patients with rheumatoid arthritis but not in that of normal persons. This substance was identified as 3-hydroxyanthranilic acid (3-HAA).

A method is described for the measurement of 3-HAA in urine, employing ether extraction, paper chromatography in a formate solvent, and visual assessment by fluorescence in ultra-violet light and colour with Ehrlich's reagent.

Normal persons excreted from less than 125 to $375 \mu \mathrm{g}$./day (average rather lower than $200 \mu \mathrm{g}$./ day); those suffering from rheumatoid arthritis excreted from less than 125 to $2,250 \mu \mathrm{g}$./day (average $820 \mu \mathrm{g}$./day).

The excessive excretion of 3-HAA in persons with rheumatoid arthritis was investigated in terms of other aspects of the activity of the disease. Some relationship was suggested between excretion levels, the erythrocyte sedimentation rate, and the sensitized sheep cell test.

Cases of osteoarthritis, other orthopaedic conditions, and miscellaneous diseases were examined. A few of the latter, including diseases of the haemopoietic system, were associated with increased excretion of 3-HAA.

It has recently been claimed that in the urine of patients suffering from one or other of the collagen diseases there may be detected a postulated but hitherto unisolated intermediate of tyrosine metabolism, namely, 2,5-dihydroxyphenylpyruvic acid (Nishimura, Shibata, Yasui, and Okamoto, 1956). This substance was recognized by its purple fluorescence in ultra-violet light after paper chromatography. It was characterized by reduction of ammoniacal silver nitrate and Brigg's reagents and by reactions with ferric chloride, Fenton's and hydrazine reagents. Its chromatographic behaviour in two solvent systems and its ultra-violet absorption spectrum were found to be the same as those of a synthetic specimen of 2,5-dihydroxyphenylpyruvic acid. In the system

\footnotetext{
* Present address: Department of Bacteriology, Edinburgh Royal Infirmary.
}

n-butanol:acetic acid:water $(4: 1: 1)$ two spots were obtained, depending on whether the urine was acid or neutral during its concentration ; one of the spots was thought to be the quinone derivative of 2,5-dihydroxyphenylpyruvic acid and was seen also in chromatograms of the synthetic substance.

In 38 cases of collagen disease the urine contained the substance described. It was absent from the urine of normal persons, those suffering from a variety of other diseases, or unaffected relatives of patients with collagen disease. The authors concluded that a fundamental biochemical clue to the aetiology of collagen diseases had been found, and that these diseases must be grouped with phenylketonuria, alcaptonuria, tyrosinosis, and albinism in that they are caused by an inherent error in the metabolism of tyrosine. 


\section{Examination of Urine for 2,5-Dihydroxy- phenylpyruvic Acid}

The Japanese workers' description of the preparation of urine extracts for chromatography was limited to the following paragraph:

"Either as it was or acidified with $10 \% \mathrm{H}_{2} \mathrm{SO}_{4}$, the 24-hr. urine of diffuse collagen disease patients was concentrated till about $20 \mathrm{ml}$. of it remained, and after another further treatment of it with $60 \%$ alcohol, and the resultant elimination of inorganic salts, the urine thus obtained was subjected to experiment. Then one-dimensional paper chromatography was performed."

In an attempt to follow the above procedure $250 \mathrm{ml}$. of urine (freshly collected in acid to give a final $p H$ of 3) from a case of acute rheumatoid arthritis was distilled at reduced pressure under nitrogen, the external temperature being kept below $40^{\circ} \mathrm{C}$. until a few millilitres of viscous residue remained. This was taken up in alcohol to a concentration of $60 \%$ and centrifuged. The supernatant was subjected to chromatography overnight on Whatman No. 1 paper by the ascending technique using the solvent system specified by the Japanese workers (n-butanol:acetic acid: water $4: 1: 1)$. When viewed under ultra-violet light ("hanovia" medium pressure quartz arc with Wood's glass filter or "hanovia" chromatolite $\mathrm{Hg}$ resonance lamp $2537 \AA$ ) a large number of fluorescent areas was detectable and subsequent spraying with ammoniacal silver nitrate solution $\left(0.1 \mathrm{~N} \mathrm{AgNO}_{3}\right.$ $25 \mathrm{ml}$., ammonia S.G. $0.888 \mathrm{ml}$., water to $50 \mathrm{ml}$.) revealed a number of reducing substances. However, no fluorescent reducing substance was seen which had the $\mathbf{R}_{\mathbf{F}}$ value stipulated by Nishimura and his colleagues. Furthermore, and perhaps of especial importance in view of the number of closely related compounds into which the quinol sought might be transformed, no difference was detectable in urine from normal individuals and rheumatoid patients when chromatography was carried out in the butanol system or in phenol:water: $\mathrm{H}_{2} \mathrm{SO}_{3}$, a solvent more suitable than the former for the preservation of a highly oxidizable substance (McMillan, 1957).

An attempt was made to purify the urine before chromatography by adsorption upon alumina (McMillan, 1957). A readily available quinol derivative fairly closely related to that being sought, namely, 2,5-dihydroxybenzoic acid (gentisic acid), was added to whole urine in amounts of $1 \mu \mathrm{g} . / \mathrm{ml}$. The quinol remained in the filtrate during alumina treatment whereas under identical conditions the compound 3,4-dihydroxyphenylacetic acid was adsorbed and none was detected in the filtrate. In neither the eluate nor the filtrate of urine from a case of active rheumatoid arthritis could a fluorescent reducing substance be found to correspond with the Japanese description.

When fresh urine, collected in acid, was extracted with ether at $p \mathrm{H} 3$ and the ether-soluble portion chromatographed, a fluorescent reducing substance was conspicuous in the rheumatoid specimens but not in those from normal persons. The ether extracts were distilled to dryness at reduced pressure at a temperature not exceeding $40^{\circ} \mathrm{C}$., and the residue, which was derived from $250 \mathrm{ml}$. urine, was taken up in $1 \mathrm{ml}$. ethanol. Upon chromatography, $0.02 \mathrm{ml}$. of this solution gave an easily visible purple spot when viewed under ultra-violet light and had an $\mathbf{R}_{F}$ of 0.84 in the butanol system and 0.61 in the phenol system. The former $\mathbf{R}_{\mathbf{F}}$ is higher than either of the values quoted by the Japanese workers for 2,5-dihydroxyphenylpyruvic acid or its quinone.

\section{Identification of the Fluorescent Reducing Substance in Urine from Rheumatoid Patients as 3-Hydroxyanthranilic Acid}

The extract described above was chromatographed in the butanol system and the paper was sprayed with a variety of colour-producing reagents with the following results at the site of fluorescence :

Ammoniacal Silver Nitrate. - Dark grey ; reduction occurred in the cold and preceded that of any other substance present elsewhere on the paper.

Gibbs Reagent (2,6-Dichlorquinone Chloroimide $0.2 \% \mathrm{w} / \mathrm{v}$ in Ethanol).-(1) Reagent alone, a purple colour exclusive to the fluorescent spot developed slowly. (2) The reagent diluted with an equal volume of $0.66 \mathrm{M}$ phosphate buffer at pH 7.0 gave an immediate purple colour with various colours appearing later in neighbouring areas. (3) The reagent followed by saturated sodium bicarbonate solution or borate solution gave an immediate blue colour at the fluorescent spot, quickly followed, especially in the case of borate, by an array of colours obscuring the original single spot.

Ferric Chloride $(0.2 \% \mathrm{w} / \mathrm{v}$ in Water). $-\mathrm{A}$ faint yellow colour appeared.

Potassium Ferricyanide (6.6\% Aqueous Solution in Phosphate Buffer at $p H$ 5.9). - This gave a pink colour.

Potassium Iodate $(1 \% \mathrm{w} / \mathrm{v}$ in Water).-This gave a grey colour.

Fenton's Reagent (Nishimura, Yasui, Okamoto, Kanazawa, Kotake, and Shibata, 1958).-This gave a brown colour.

Ehrlich's Reagent.-This is made up of p-dimethylaminobenzaldehyde, $10 \% \mathrm{w} / \mathrm{v}$, in concentrated $\mathrm{HCl}$ solution, 1 vol., acetone, 4 vol., mixed immediately before use, and gave a slowly developing distinct orange-yellow colour.

The following reagents either gave no colours at all in the appropriate area or else gave colours which did not properly coincide with the fluorescent spot: Diazotized sulphanilic acid, p-nitraniline 
and p-anisidine respectively, ninhydrin, nitrosonaphthol reagent, xanthydrol reagent, Altman's reagent, 2,4-dinitrophenylhydrazine (for all the preceding reagents see Smith, 1958), Millon's reagent, Brigg's reagent (Nishimura et al., 1958), preceding reagents (see Smith, 1958), Millon's Roux reagent (Roux, 1951) and bromothymol blue-acidified potassium iodide-starch ammonia (as used for homogentisic acid by Consden, Forbes, Glynn, and Stanier, 1952). The fluorescent substance was seen to develop a brown colour when the papers were exposed to the atmosphere for an hour or two ; it was unstable in alkaline solution, a $p \mathrm{H}$ of 9 causing appreciable loss.

By consideration of the above properties and by comparing the observed $\mathbf{R}_{F}$ in various solvent systems with those published for phenolic acids known to occur in urine (for references see Smith, 1958), it appeared probable that the fluorescent substance was 3-hydroxyanthranilic acid. A specimen of this substance (Roche) proved to have the same fluorescence under both ultra-violet light sources and to give the same colour reactions as the unknown substance. When added to the unknown substance in a urine concentrate it had an identical $\mathbf{R}_{F}$ in four solvent systems, namely, 0.61 in phenol : water: $\mathrm{H}_{2} \mathrm{SO}_{3} ; 0.84$ in n-butanol: acetic acid: water $(4: 1: 1), 0.52$ in benzene : acetic acid:water $(125: 72: 3)$, and 0.63 in sodium formate: formic acid:water $(10 \mathrm{~g}$.: $1 \mathrm{ml}$.: 200 mil.). Both substances were largely destroyed in the system iso-propanol:0.88 ammonia: water (200:10:20).

\section{Semiquantitative Measurement of the Amount of 3-Hydroxyanthranilic Acid in Urine}

The fluorescence of 3-hydroxyanthranilic acid (3-HAA) in ultra-violet light could be detected easily on the chromatogram paper in amounts as low as $0.25 \mu \mathrm{g}$., whereas none of the colour reactions was as sensitive as this. Chromatography in the butanol system was unsuited to quantitative work because very many phenolic acids have a similar high $R_{F}$ value; urine extracts prepared by simple ether extraction gave neighbouring spots which were highly fluorescent under ultra-violet light, and, though they could be distinguished from 3-hydroxyanthranilic acid by their blue colour, they partially, or sometimes completely, obscured the purple colour of 3-HAA. The benzene system was unsuitable because the spots became too spread out. Both the phenol and formate systems gave sharply defined spots and some urine extracts could be satisfactorily assessed in terms of standard amounts of 3-HAA run on the same sheets of paper. Many, however, showed one or more fluorescent substance which interfered more or less seriously with such a comparison. Two-dimensional chromatography, employing the phenol system followed by the formate, failed to eradicate the interference. After the use of any of the above solvent systems colour development on the paper with Ehrlich's reagent or buffered potassium ferricyanide solution proved insufficiently sensitive and ammoniacal silver nitrate or Gibbs reagent at various $p \mathbf{H}$ values insufficiently specific.

Owing to the presence of a basic group, 3-HAA is not extracted by ether from highly acid aqueous solution. When urine was acidified with one-tenth of its volume of concentrated $\mathrm{HCl}$ and extracted with ether, the concentrated ether extracts on chromatography usually contained much material fluorescent in ultra-violet light, whereas subsequent extraction of the aqueous residue after adjustment of the $p H$ to 3 gave a preparation which frequently showed only one fluorescent area, that due to 3-HAA. These findings were applicable to chromatography in the phenol, formate, and butanol systems. At each $p \mathrm{H}$ ether was extracted with four equal portions whose total volume equalled that of the aqueous phase. Experiments showed that, when added to the urine in amounts as high as $1 \mu \mathrm{g}$. $/ \mathrm{ml}$., 3-HAA could be satisfactorily accounted for during the processes of extraction, concentration, and chromatography; the four combined extracts made at $p \mathrm{H} 3$ contained as far as could be assessed by visual comparison the whole of the added 3-HAA, whereas the four ether extracts of the highly acid urine and two additional extracts made at $p H 3$ subsequent to the four normally prepared showed no trace of 3-HAA.

After such a procedure of double ether extraction some urine extracts continued to give additional fluorescent spots. Commonly there was found a blue spot immediately above the purple 3-HAA in both phenol and formate systems; the fluorescence of this substance was much greater under the "hanovia" $2537 \AA$ lamp than under the Woods glass whereas that of 3-HAA was similar under both, so that there was no danger of confusion. In order to guard against the possibility of interference at the actual site of 3-HAA, though this appeared unlikely, after assessment under ultra-violet light the chromatogram was always sprayed with Ehrlich's reagent and some hours later the intensities and sizes of the yellow spots were compared with those of the standards. Amounts less than $0.5 \mu \mathrm{g}$. usually did not show up, but for amounts greater 
than this there was never any great discrepancy between the values obtained by the two methods of comparison. Chromatograms developed in the phenol system were easily assessed by fluorescence in ultra-violet light and Ehrlich colour (pink), the paper being freed of phenol by benzene extraction followed by hanging in a current of warm air for two to three hours. In fact, in $80 \%$ of the assays to be reported duplicate chromatograms were thus run; there was always close agreement with the standard method using formate. After double ether extraction some chromatograms run in the butanol system and dried in warm air were sprayed with Gibbs' reagent followed by saturated sodium dihydrogen phosphate solution. This method of assessment was quite unsatisfactory as the initial spots of 3-HAA were rapidly obscured by several other closely situated substances (cf. Tompsett, 1959).

It was found that urine acidified to $p \mathrm{H} 3$ or thereabouts maintained its content of 3-HAA for many days when stored in the refrigerator. The ether extracts were unstable and were always assayed as soon as possible, usually on the day of preparation. Similarly the developed chromatograms were always assessed as soon as the papers were dry.

The possibility of completing the assay of 3-HAA by spectrofluorometric measurement was not investigated in this study.

\section{Method}

Preparation of Extract.--Urine was collected for 24 hours, $15 \mathrm{ml}$. of $10 \% \mathrm{H}_{2} \mathrm{SO}_{4}$ being used as preservative. To a filtered aliquot measuring $10 \%$ of the whole specimen was added one-tenth of its volume of concentrated $\mathrm{HCl}$. The mixture was then shaken for two-minute periods with an equal volume of peroxidefree ether divided into four equal portions. Centrifugation was usually necessary to effect separation. After each extraction the ether layer was pipetted off and discarded. The $p \mathrm{H}$ of the aqueous phase was then adjusted with a volume of $10 \mathrm{~N} \mathrm{NaOH}$ equiva'ent to that of the concentrated $\mathrm{HCl}$ previously used, followed by more dilute alkali until a $p \mathrm{H}$ of 3.5 was reached (B.D.H. 2540 indicator paper). Ether extraction was repeated with the same amounts as before. The four resulting ether fractions were combined and distilled to dryness at reduced pressure under nitrogen at a bath temperature not exceeding $40^{\circ} \mathrm{C}$. The residue was taken up in four small successive amounts of ethanol and the solution was evaporated to a volume of $0.5 \mathrm{ml}$. This pale, strawcoloured solution was used.for chromatography.

The general chromatographic technique was that described by McMillan (1957). The paper was marked out into four strips (each $2 \mathrm{~cm}$. wide) for the urine extract (usually $0.01,0.005,0.0025$, and $0.00125 \mathrm{ml}$.) and four for the standard 3-hydroxyanthranilic acid solution $(2.0,1.0,0.5$, and $0.25 \mu \mathrm{g}$.). Before use the standard was diluted 1 in 5 in ethanol from a stock refrigerated solution containing $20 \mathrm{mg}$. $/ 20 \mathrm{ml}$. ethanol to which $0.05 \mathrm{ml}$. of concentrated $\mathrm{HCl}$ had been added. The solvent (sodium formate $10 \mathrm{~g}$., water $200 \mathrm{ml}$., formic acid $1 \mathrm{ml}$.) was renewed on each occasion. Two or three sheets of paper were hung in the same tank for two to three hours until the solvent front reached about $25 \mathrm{~cm}$. A sheet of sponge rubber and a wrapper of cellophane paper were applied to the underside of the lid of the tank which was then quickly sealed by pressure from above (F. Albert-Recht, personal communication). The sheets were dried in the air at room temperature using a hair dryer. The amount of 3-hydroxyanthranilic acid in each of the four urine extract chromatograms was assessed visually by transmitted light from both the ultra-violet lamps previously described. The paper was then sprayed with Ehrlich's reagent and further comparisons made after about 18 hours.

\section{Results}

The excretion of 3-hydroxyanthranilic acid by 83 persons was determined. Twenty-five patients suffering from rheumatoid arthritis (all of whom were in-patients of the Rheumatic Unit, Northern General Hospital, Edinburgh) excreted quantities ranging from less than $125 \mu \mathrm{g}$. (the minimal amount detectable by the method) to $2,250 \mu \mathrm{g}$. i day, the average figure being $820 \mu \mathrm{g}$./day. In a few instances excretion was measured on more than one occasion, when values of the same order were found. The figures bore no relationship to the age or sex of the patient. The patients had received no steroid therapy or other drugs for at least three days before the urine was collected.

Twelve healthy laboratory workers excreted from less than 125 to $375 \mu \mathrm{g}$./day, the average being rather lower than $200 \mu \mathrm{g}$./day. Fig. 1 shows that whereas only two of these normal persons excreted more than $250 \mu \mathrm{g}$./day, all rheumatoid subjects save three excreted $375 \mu \mathrm{g}$. or more/day. There would therefore appear to be some considerable difference between these two groups. One normal person who received heavy doses of either aspirin or panadol for three days showed the same low excretion three days later.

Analysis of the group of rheumatoid patients failed to show any correlation between the level of excretion of 3-hydroxyanthranilic acid and some of the criteria which might be used to assess the severity of the disease, namely, the duration of symptoms, the extent of tissue damage as shown radiologically, or the degree of anaemia. Comparison of the erythrocyte sedimentation rate (E.S.R.) measured by the Westergren method with excretion of 3-hydroxyanthranilic acid 


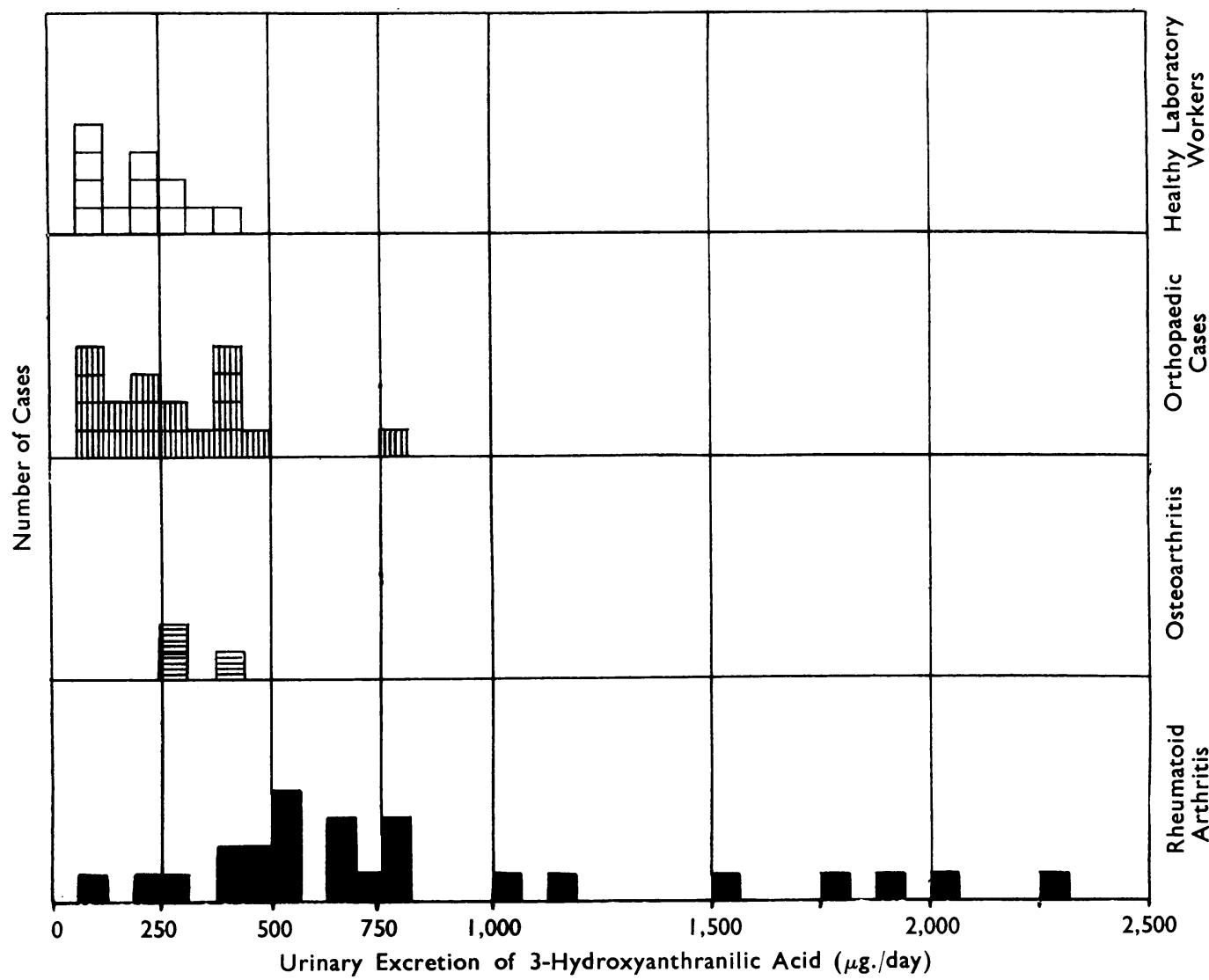

FIG. 1.-Urinary excretion of 3-hydroxyanthranilic acid in rheumatoid arthritis, osteoarthritis, and orthopaedic conditions as compared with normal persons.

showed that the cases fell into two groups (Fig. 2). The first comprises the seven highest excretors, that is, all who excreted $1,000 \mu \mathrm{g}$. or more per day, who had E.S.R. values ranging from 27 to $86 \mathrm{~mm}$./hour (average $61 \mathrm{~mm}$./ hr.). The E.S.R. of the remaining 18 cases ranged from 3 to 54 (average 32 ) $\mathrm{mm}$./ hr. and in these the 3-hydroxyanthranilic acid excretion never exceeded $750 \mu \mathrm{g}$./day. There is thus a trend towards proportionality between the two values. Comparison with the titres obtained in the sensitized sheep cell test (for details see Duthie, Brown, Knox, and Thompson, 1957) showed a similar pattern of distribution as obtained for the E.S.R. for the seven highest excretors, all of whose titres were positive, ranging from $1: 128$ to $1: 2,048$ (Fig. 3). The remaining 18 cases, however, had titres from $1: 8$ to $1: 2,048$. Thus all patients with a high excretion of 3-hydroxyanthranilic acid gave a positive sensitized sheep cell test, but a positive titre was not necessarily accompanied by a high level of excretion. Degrees of rheumatoid activity have been classified by Duthie et al. (1957) in terms of E.S.R., haemoglobin levels. joint involvement, and systemic disturbance. The activity of the disease of some of the above patients was independently assessed by the former workers. Three cases were allocated to group 1 (inactive), 18 to group 2 (moderately active), and none to group 3 (very active). No relationship with the level of excretion of 3-hydroxyanthranilic acid could be discerned. Similarly, by the same authors' classification of functional capacity, one case fell in group 1 (no disability), 14 in group 2 (moderate incapacity), three in group 3 (marked incapacity), and one in group 4 (confined to bed or chair); again no correlation with excretion could be found.

A small group of patients with osteoarthritis was investigated. These patients were receiving similar basic treatment and diet in the same wards and at the same time as the rheumatoid group, 


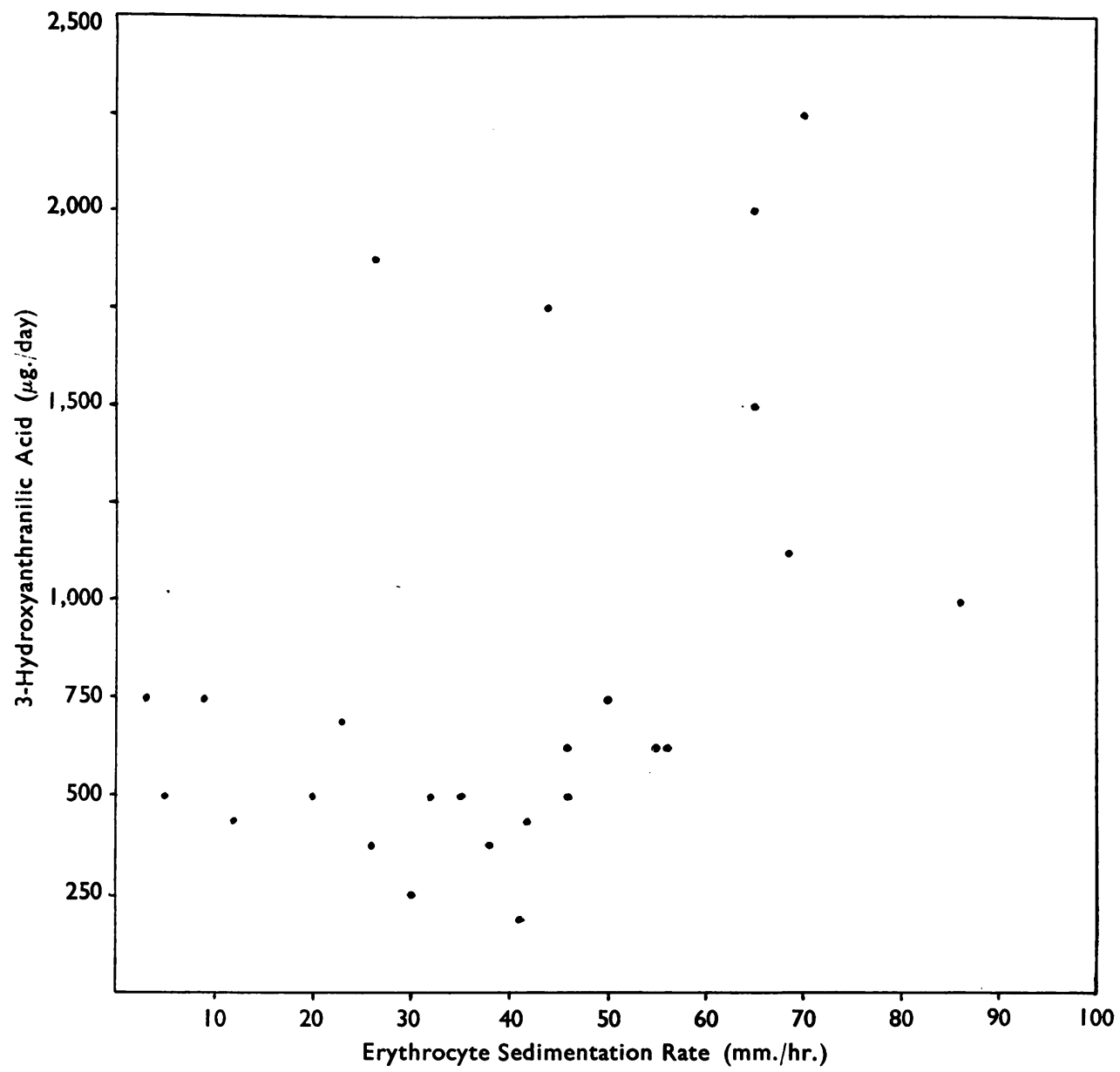

FIG. 2.-The relationship between urinary excretion of 3-hydroxyanthranilic acid and the erythrocyte sedimentation rate in patients suffering from rheumatoid arthritis.

but 3-hydroxyanthranilic acid excretion showed values not in excess of those of normal persons (Fig. 1). A larger group of 18 orthopaedic cases was studied. Of these patients, some were ambulant at the time of examination, some were more or less immobilized. Fig. 1 shows that in these cases the level of excretion of 3-hydroxyanthranilic acid was similar to that of normal persons, the average being $240 \mu \mathrm{g}$./day; with a single exception the range extended from less than 125 to $440 \mu \mathrm{g}$./day. A wide range of traumatic and non-traumatic conditions was represented; there was no obvious relationship between excretion levels and such factors as extent of tissue injury or degree of immobilization. The exceptional patient who excreted as much as 750 $\mu \mathrm{g}$./day was one who 15 weeks previously had undergone fusion of the hip on account of an old injury.

In a miscellaneous group (Fig. 4), one case of ankylosing spondylitis showed a low excretion, as did a single case of disseminated lupus erythematosus receiving prednisolone. In two cases of psoriatic arthritis the excretion was low in one and very high in the other $(1,750 \mu \mathrm{g}$./day). Two patients who had undergone partial gastrectomy two days before the collection of urine showed a low excretion.

In medical conditions such as myocardial infarction, pulmonary infarction, chronic nephritis, uraemia, hepatic cirrhosis, duodenal ulcer, etc., excretion figures were uniformly low. This was the rule also with patients having sepsis or anaemia, including cases of staphylococcal 


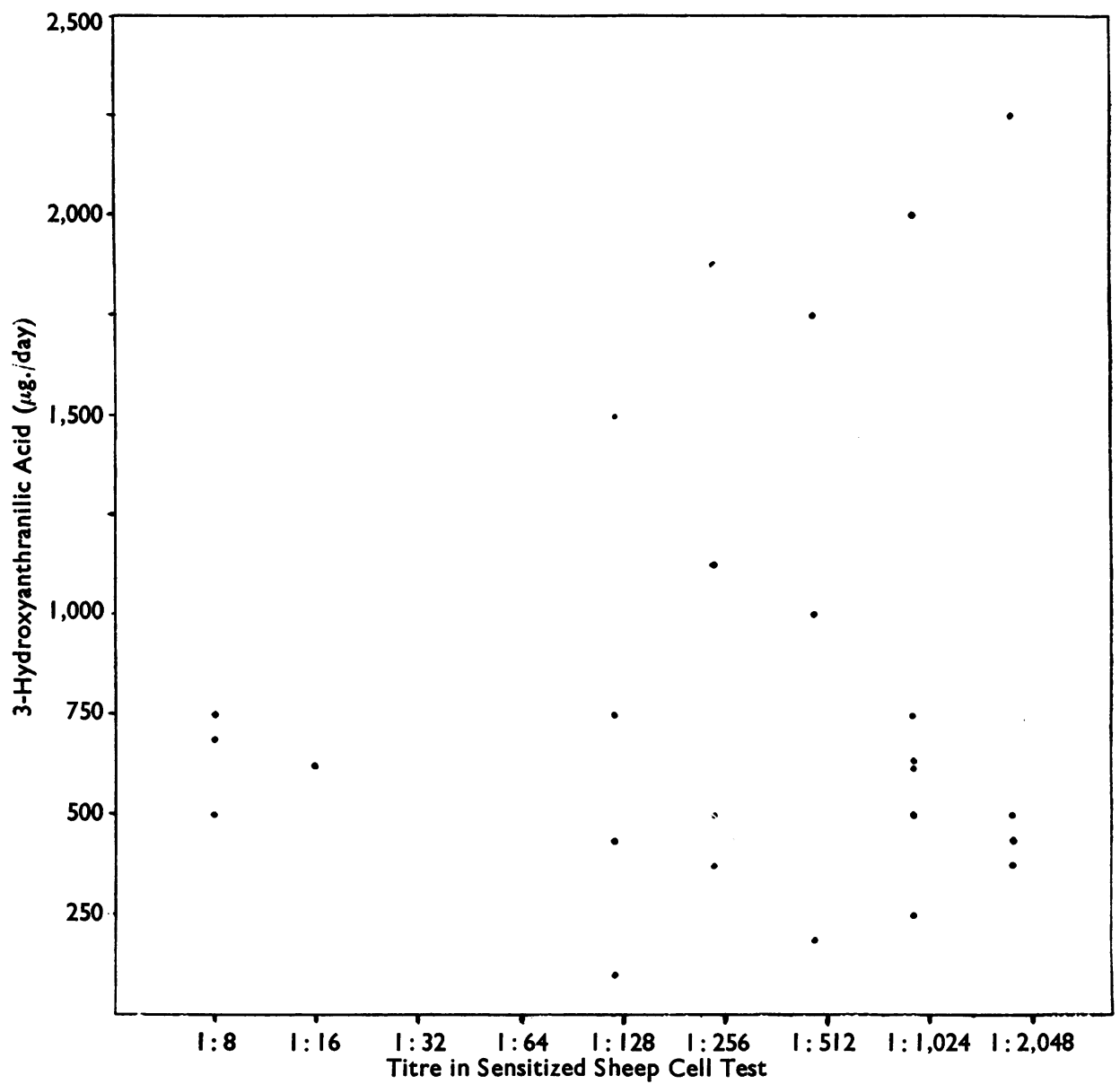

Fro. 3.-The relationship between the titre obtained in the sensitized sheep cell test and the urinary excretion of 3-hydroxyanthranilic acid.

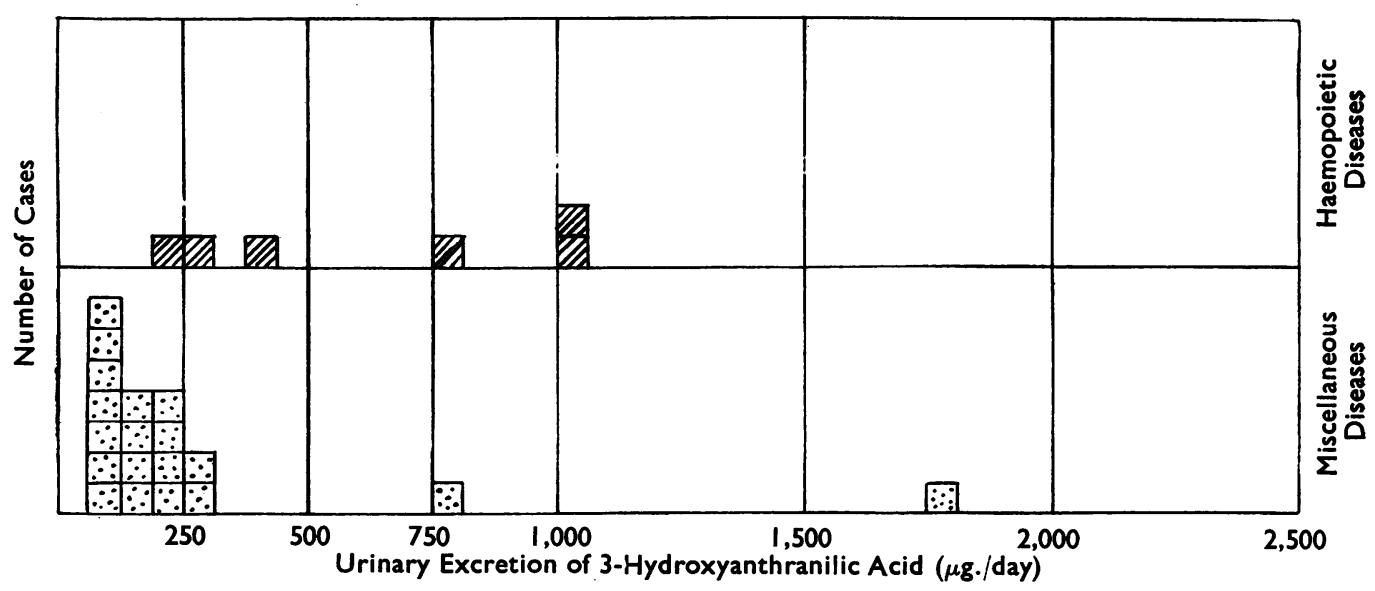

FIG.4.-Urinary excretion of 3-hydroxyanthranilic acid in diseases of the haemopoietic system and in other miscellaneous conditions. 
pneumonia and active pulmonary tuberculosis. Fever alone caused no rise. In view of the possible relationship between the E.S.R. and the excretion of 3-hydroxyanthranilic acid in rheumatoid arthritis special attention was paid to patients having a very high E.S.R. from other causes. All gave low figures with the exception of one patient with myelosclerosis with aplastic anaemia who had an E.S.R. of $125 \mathrm{~mm}$./hour and an excretion of 3-hydroxyanthranilic acid as high as $1,000 \mu \mathrm{g}$./day. The other five patients having blood diseases included in this survey also showed a tendency to raised outputs. Thus three patients with chronic myeloid leukaemia, whose white cell counts were $209,000,182,000$, and $60,800 /$ c.mm. respectively, excreted 250,750 , and $1,000 \mu \mathrm{g} . /$ day ; the second of these had undergone no treatment, the other two were receiving irradiation therapy. Two other patients suffering from megakaryocytic leukaemia and myelomatosis, whose white cell counts were not raised, excreted 190 and $440 \mu \mathrm{g}$./day respectively.

\section{Discussion}

This study fails to substantiate the claim of Nishimura et al. that 2,5-dihydroxyphenylpyruvic acid is excreted in the urine by patients with rheumatoid arthritis. The data published by the Japanese workers were incomplete, but an approximation to their method of identification failed to demonstrate any such compound. In the absence of excretion of 2,5-dihydroxyphenylpyruvic acid there is no evidence for the hypothesis that the collagen diseases are due to an inherent error in the metabolism of tyrosine.

Chromatographic examination of the urine in cases of rheumatoid arthritis for a strongly reducing substance has shown, however, that free 3-hydroxyanthranilic acid is excreted in increased amount as compared with normal subjects. The compound is known to be a normal metabolite of tryptophan; it is the immediate degradation product of 3-hydroxykynurenine and a precursor of nicotinic acid. It has so far been of limited medical interest, mainly as a possible carcinogen. Since the suggestion that o-aminophenols were carcinogenic (Bonser, Clayson, Jull, and Pyrah, 1954), attention has been drawn to the two naturally occurring compounds, namely, 3hydroxykynurenine and 3-hydroxyanthranilic acid, which have this structure. In fact, 3hydroxyanthranilic acid was shown to produce cancer of the bladder in mice (Watson, 1956) and to be excreted in the unconjugated state in increased amount in human cancer of the bladder (Boyland and Williams, 1956). The latter authors, who used a method involving adsorption on charcoal and gradient-elution chromatography, give the normal excretion of free 3-hydroxyanthranilic acid as 8 to 32 (average 20) $\mathrm{mg}$./10 1 . urine; this is considerably higher than the values reported in the present paper.

Dalgliesh and Tekman (1954) reported an increased urinary output of kynurenine and 3hydroxykynurenine in half of a series of febrile patients, suggesting it was due to increased tissue breakdown; loss of weight without fever and thyrotoxicosis did not have this effect. Musajo, Benassi, and Parpajola (1956) reported that recession of fever did not arrest the excessive excretion of kynurenines. In a large series, the same authors (1955), using paper chromatography, found "very small amounts" of 3-HAA to be excreted by normal human subjects. In 11 out of 13 subjects with pulmonary tuberculosis excretion was definitely higher. Also in 59 out of 76 cases of leukaemia of various types, myelomatosis, Hodgkin's disease, and lymphosarcoma, considerable quantities of kynurenine and 3-hydroxykynurenine were found, but none was detectable in normal persons; no details were given in regard to 3-HAA, apart from a statement that excretion was raised in about one-third of the cases. The authors emphasized that the detection of tryptophan metabolites in human urine in the absence of special diets or administration of tryptophan was rare, and such excretion was linked with pathological conditions.

An increased excretion of 3-HAA in any of the collagen diseases has not been reported previously. In the present series of cases the magnitude of the increase would appear to bear no relation to any of the major features of the disease except perhaps the E.S.R., where there was some proportionality, and the sensitized sheep cell test, which was always positive in association with the highest excretion rates. A high E.S.R. per se does not lead to increased excretion since many non-rheumatoid patients had a very high E.S.R. without such an increase. The rheumatoid patients had received no steroid or other drugs for at least three days before 3-HAA excretion was measured. A normal person who received heavy doses of either aspirin or panadol for three days showed no increase in 3-HAA excretion three days later. The effect of drugs on excretion by the rheumatoid patients was not investigated.

In agreement with Musajo et al. (1956), no direct relationship was found between 3-HAA excretion and fever; but contrary to the above report no increase was found in active pulmonary tuberculosis. 
The raised excretion in some patients with haematological disorders is of interest and merits a further survey and investigation. Cases of erythrogenesis imperfecta have been reported to excrete abnormal amounts of anthranilic acid (Altman and Miller, 1953). In diseases of the blood-forming organs, then, it would appear that more than one tryptophan metabolite may be excreted in excess. Whether metabolites other than 3-HAA are involved in rheumatoid arthritis has not been determined and there is no evidence to suggest that the findings in rheumatoid arthritis are in any way related to those in blood disease.

An increase in urinary 3-HAA could be due to increased tryptophan catabolism. However, many patients in whom tissue was probably wasting failed to show this effect and in rheumatoid arthritis itself there was no correlation with such wasting. Possibly 3-hydroxyanthranilic acid could accumulate as a result of enzymic imbalance such as can be produced in vivo in the case of certain other tryptophan metabolites by vitamin deficiency. Further insight into the abnormality in rheumatoid arthritis might be gained by studies along these lines.

This research was supported by a grant from the Scottish Hospitals Endowment Research Trust to Dr.
C. P. Stewart and this assistance is gratefully acknowledged. I am grateful to Dr. C. P. Stewart for his critical interest; to Dr. J. J. R. Duthie for much helpful co-operation in connexion with the patients under his care at the Rheumatic Unit, Northern General Hospital, Edinburgh, and also for criticism of this paper; also to the staff of the Orthopaedic, Medical, and Radiotherapy Departments of the Edinburgh Royal Infirmary for facilities in connexion with the collection of specimens.

\section{REFERENCES}

Altman, K. I., and Miller, G. (1953). Nature (Lond.), 172, 868. Bonser, G. M., Clayson, D. B., Jull, J. W., and Pyrah, L. N. (1954). Brit. J. Urol., $26,49$.

Boyland, E., and Williams, D. C. (1956). Biochem. J., 64, 578.

Consden, R., Forbes, H. A. W., Glynn, L. E., and Stanier, W. M. (1952). Ibid., $50,274$.

Dalgliesh, C. E., and Tekman, S. (1954). Ibid., 56, 458.

Duthie, J. J. R., Brown, P. E., Knox, J. D. E., and Thompson, M. (1957). Ann. rheum. Dis., 16, 411.

McMillan, Mary (1957). Lancet, 1, 715.

Musajo, L., Benassi, C. A., and Parpajola, A. (1955). Nature (Lond.), 175,855 .

(1956). Clin. Chim. Acta, 1, 229.

Nishimura, N., Shibata, Y., Yasui, M., and Okamoto, H. (1956). Proc. Japan Acad., 32, 697.

- Yasui, M., Okamoto, H., Kanazawa, M., Kotake, Y., and Shibata, Y. (1958). A.M.A. Arch. Derm., 77, 255.

Roux, D. G. (1951). Nature (Lond.), 168, 1041.

Smith, I. (1958). Chromatographic Techniques. Heinemann, London.

Tompsett, S. L. (1959). Clin. Chim. Acta, 4, 411.

Watson, J. G. (1956). Surg. Forum, 7, 679. 